\title{
Adaptive neural control for space structure vibration suppression
}

\author{
Larry Davis $\uparrow$, David Hyland $\ddagger$, Gary Yen $§$ and Alok Das $\|$ \\ † Planning Systems, Inc., 2500 Harbor Street, Melbourne, FL 32902, USA \\ \$University of Michigan, Department of Aerospace Engineering, Ann Arbor, MI 48109, \\ USA \\ $\S$ Oklahoma State University, School of Electrical Engineering, Stillwater, OK 74078, USA \\ || USAF Research Laboratory, Space Vehicle Technology Division, Kirtland AFB, \\ NM 87117, USA
}

Received 18 May 1998, in final form 29 July 1998

\begin{abstract}
Despite recent advances in efficiency, current methodologies for space structure control design still engage significant human resources for engineering development and routine maintenance. The adaptive neural control (ANC) program is part of an effort to develop neural network based controllers capable of self-optimization, on-line adaptation and autonomous fault detection and control recovery. This development in addition supports the long-term space exploration objectives for which autonomous spacecraft involving self-reliant control systems are a necessity.

The ANC program comprises two phases. The first, basic phase focused on the development of efficient and completely autonomous neural network feedforward control for the case of broadband disturbances. Algorithms were developed that work with no prior modeling information about the system to be controlled and adapt to changing conditions, while minimizing or eliminating the introduction of extraneous training signals.

The algorithms were demonstrated experimentally on an optical structure testbed at Harris. The second phase of the program demonstrated a more complex neural controller on the advanced space structures technology research experiments (ASTREX) test facility at the Air Force Research Laboratory capable of the fault-tolerant adaptive control of multiple sensors and actuators. This system used six actuation channels of the existing ACESA struts on the ASTREX structure to simultaneously cancel three independent tonal disturbances in the $10-15 \mathrm{~Hz}$ band, measured at non-collocated sensors on the secondary tower of the structure. The system demonstrated impressive fault-recovery performance, maintaining good cancellation performance with successive actuators disabled. Cancellation of individual tones was between 25 and $55 \mathrm{~dB}$, with over $27 \mathrm{~dB}$ attenuation realized root mean square.

The algorithm required very low computational throughput, operating at a sample rate of $1 / 20 \mathrm{~Hz}$.

The results of the ANC program show that adaptive cancellation systems can reduce vibrations in precision structures without prior modeling information and can adapt successfully to certain failures in actuators or sensors, optimally reconfiguring themselves without human intervention. These capabilities should significantly reduce the expense of designing and maintaining vibration control systems for spacecraft.
\end{abstract}

\section{Introduction}

Modern engineering technology is leading to increasingly complex space structures with ever more demanding performance criteria. Currently advocated control design approaches, however, mandate a high fidelity, if not exact, dynamic model containing identified system parameters. These methodologies which call for an iterative process of finite element analysis or system identification are time consuming and computationally expensive to validate [1]. In addition, time-critical control recovery due to catastrophic failures is often left unresolved [2]. The ultimate pursuit of a higher degree of autonomous behavior that provides constant health monitoring and fault tolerance for structure platforms with minimum human intervention has high priority in order to achieve a successful flight mission $[3,4]$. To increase the ability to accommodate anticipated as well as unexpected dynamic variations in the complex interrelated components of structure platforms, a totally autonomous neural control system based solely upon onboard sensor instrumentation is actively pursued [5-7]. The work reported here is part of an effort to analytically design and experimentally validate an innovative adaptive controllers based on neural network technology that is capable of self-optimization, on-line adaptation, autonomous fault detection and controller reconfiguration. The advances achieved in adaptive neural control (ANC) has translated into greater cost-effectiveness for control design, implementation and verification and a significant expansion of DoD and commercial applications. 


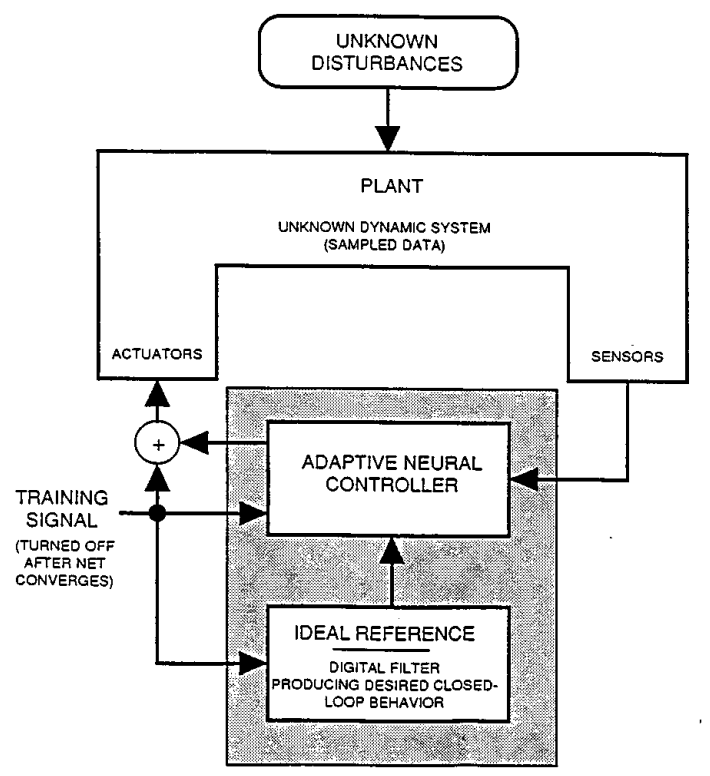

Figure 1. ANC executes simultaneous system identification and adaptively optimized control.

The near term development of adaptive systems for control is made possible by the rapid growth in pure and applied neural network research that has occurred in the last decade $[8,9]$. Much of previous work in adaptive control via neural networks concerned highly nonlinear but low dimensional systems [10-12]. In contrast, the ANC architecture developed in [13-15] concentrates on neural schemes particularly geared to structural vibration control problems. These problems involve high-order systems exhibiting very broadband dynamics. As indicated in figure 1, the ANC system combines tapped delay lines with static neurons to perform on-line system identification and adaptive control. The system adapts in the presence of unknown, persistent plant disturbances and instrumentation noise and requires no detailed prior modeling information.

There are several key features of this neural architecture that have made it particularly attractive. First, although the architecture can be visualized as a neural network, the control scheme is fundamentally a massively parallel, decentralized adaptive control algorithm that need not be implemented literally as a collection of artificial neurons. Secondly, these 'neural' algorithms feature learning capability that is distributed down to the smallest computational unit. Decentralization (distributed learning) imparts the ability to autonomously recover from hardware failures, including damage to the neural processor itself. A third key feature is that the basic neural building blocks are hierarchically organized into a set of standardized modules. Analogous to a 'Lego set', modules can be combined to build an enormous variety of systems and permit complex systems to be built up from simpler components in a transparent way. Finally, modularity and parallelism yield implementation flexibility. Specialized hardware is not required for implementation of the developed ANC architecture. The entire identification or control algorithm can be distributed among several parallel processors, and hardware suitable for this purpose is currently

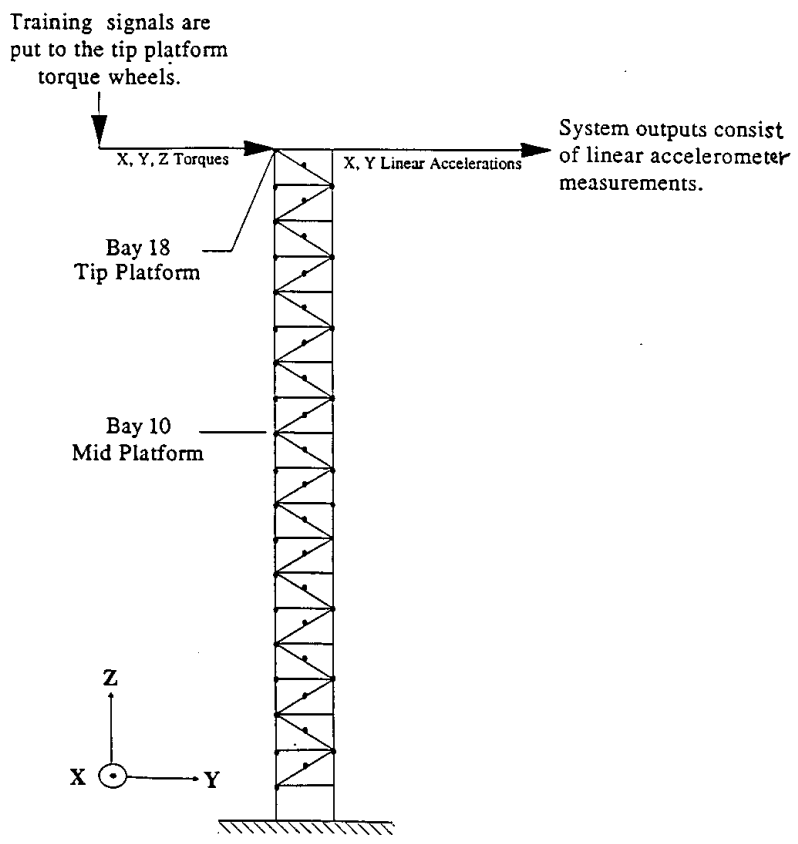

Figure 2. NASA/LaRC Mini-MAST test facility.

available and is being used for engineering development. This means that we can progress in orderly fashion from the use of existing processors to ultimately dedicated neural VLSIs $[16,17]$, thereby building our capabilities gradually and systematically.

We will present in section 2 a brief overview of our efforts dedicated to this technology and in section 3 we describe a new multi-input multi-output (MIMO) controller for tonal disturbances that is capable of optimal controller recovery following actuator or sensor failures. The capabilities of this control system are demonstrated by experimental results obtained on the ASTREX test facility located at the USAF Research Laboratory (formerly Phillips Laboratory). This will be followed by the concluding remarks in section 4 .

\section{ANC for system identification and adaptive control}

The ANC modular architecture is a set of building blocks and rules for combining them so as to achieve massively parallel and decentralized identification and adaptive control [13, 14]. The hierarchy starts, at the lowest level, with tapped delay lines and individual neurons. This is fundamentally a twoway device with a forward and backward signal path. The basic neuron is the starting point in defining the hierarchy of modular structures. The key to applying such neurons to dynamic system identification is to organize them into larger building blocks, the dynamic ganglia. A ganglion is an array of neurons allocated to establish temporal ordering within the network so as to process time histories of signals. Ganglia are interconnected by bundles of synapses, which are sometimes constrained so that their weights form Toeplitz matrics [18]. Toeplitz synapses, characterized by upper triangular weight matrices, are designed to preserve temporal ordering and causality within the network. With the above principles of 


\section{Comparison of:}

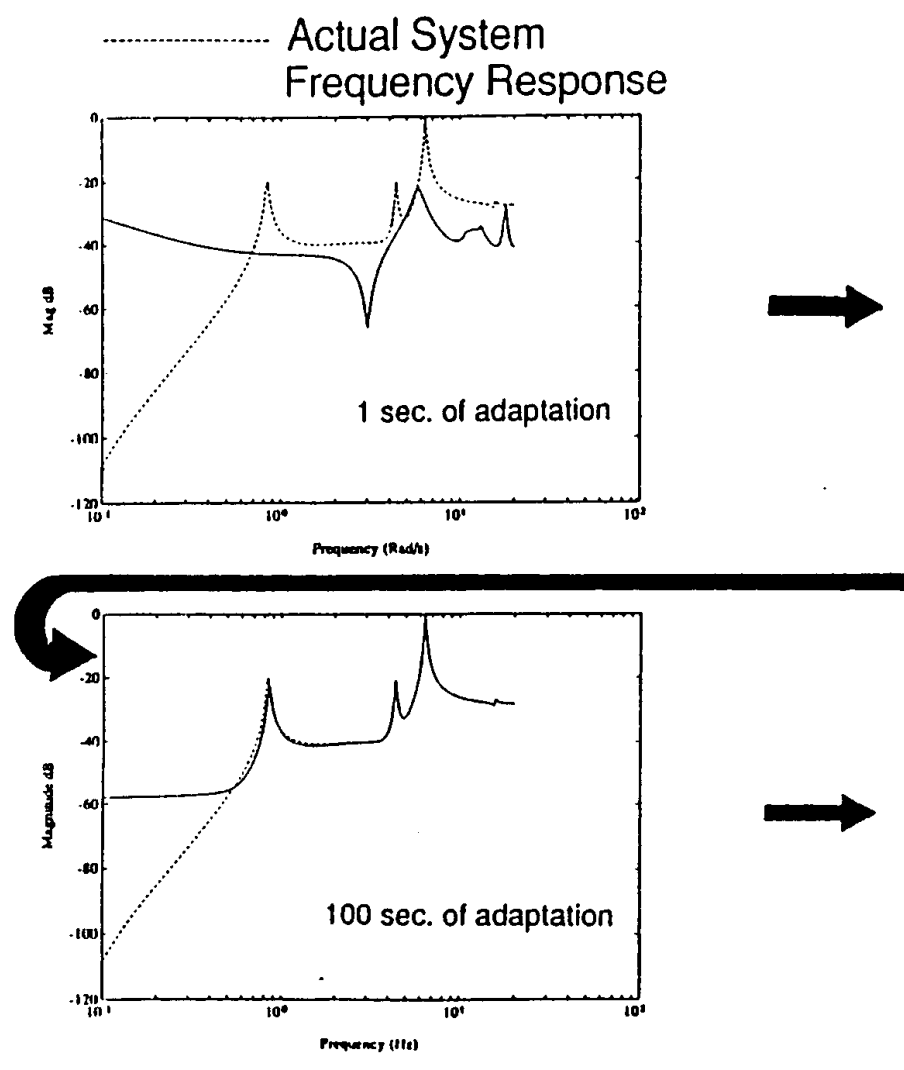

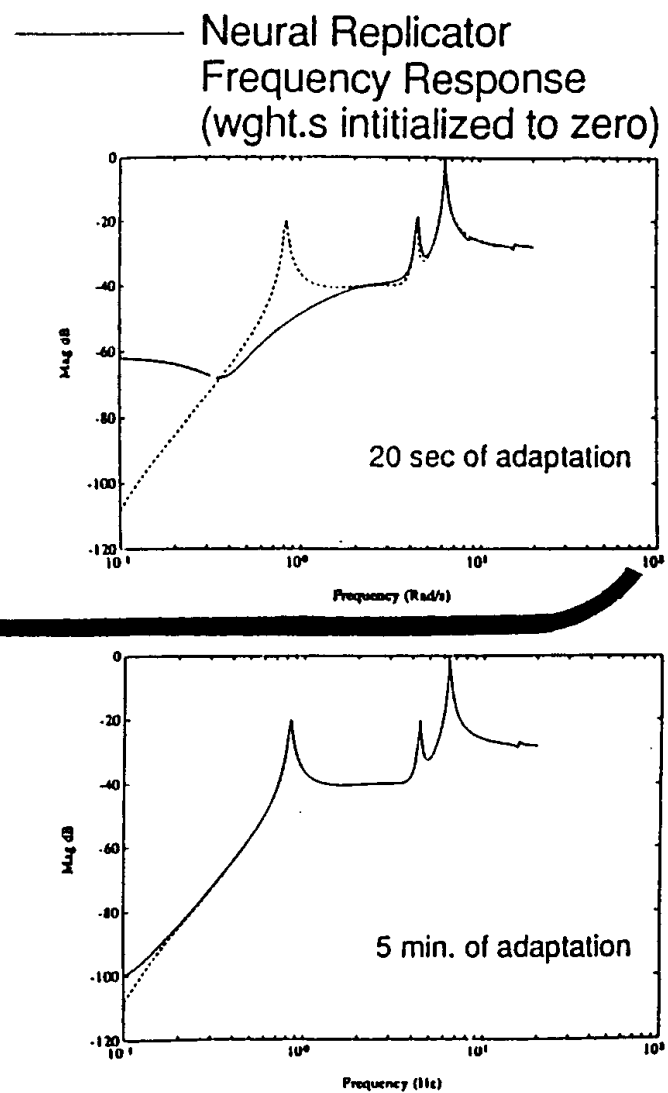

Figure 3. Mini-MAST replicator for system identification.

Toeplitz networks in mind, we can describe the next level in the structural hierarchy, namely the replicator network. Replicator units are composed of several dynamic arrays and represent the simplest functional configuration of Toeplitz networks. The fundamental task of a replicator is to duplicate the output of a previously unknown sampled data dynamic system when both replicator and system are stimulated by the same training inputs. Thus the replicator is the basic module for system identification. Several forms of replicator units are available depending on whether the system to be replicated is known to be linear, nonlinear, FIR, IIR, time-domain or frequency-domain. The work in identification methods using systems observer Markov parameters led to discovery of a new model form for dynamic systems, the ARMarkov model, so-called because it combines features of impulse response (Markov parameters) with ARMA (auto regressive moving average) models [19, 20].

The replicators are fundamentally time-domain representations of dynamic systems. For linear systems, particularly, all time-domain forms can be generalized to frequency domain or mixed time/frequency domain forms. Generally, one obtains a frequency-domain version of a particular time-domain replicator by replacing the time series inputs or the time series error signals by the outputs of fast Fourier transform (FFT) filters having these signals as inputs. This operation is equivalent to inserting a multiplication by a unitary matrix within the replicator structure. However, this superficially trivial change allows the replicator to address frequency-weighted output matching and direct frequency response identification. To illustrate the operation of a neural replicator, consider an example involving the NASA/LaRC Mini-MAST testbed [21,22]. The Mini-MAST as shown in figure 2 is a vibration control facility in the form of a 60 feet high truss beam cantilevered to the floor. Instrumentation includes torque wheel actuators at the top platform to provide control torques and accelerometers to measure lateral vibration. In this example the data acquisition system is operated at $40 \mathrm{~Hz}$ sampling rate. Training signals are inputs to the torque wheels and the system outputs consists of the linear accelerometer measurements. In this numerical simulation, we use a 17-state model of the Mini-MAST which was derived from actual test data. The system replicator's task is to duplicate the torque wheel-to-accelerometer transfer functions (that characterize the testbed for purposes of control design). We initialize the weight matrices at zero and set the learning rate to 1.0. Figure 3 illustrates the results obtained under these assumptions. We show a sequence of comparisons between the actual system frequency response and the neural network frequency response at various time instants during the learning process. The neural replicator is seen to progressively lock on to one vibration mode after another, starting with the dominant modes near $10 \mathrm{~Hz}$ and finishing with the lowest frequency modes near $1 \mathrm{~Hz}$. A period of $100 \mathrm{~s}$ of adaptation produces an excellent neural replica and essentially exact agreement is reached after five minutes of adaptation [23]. 


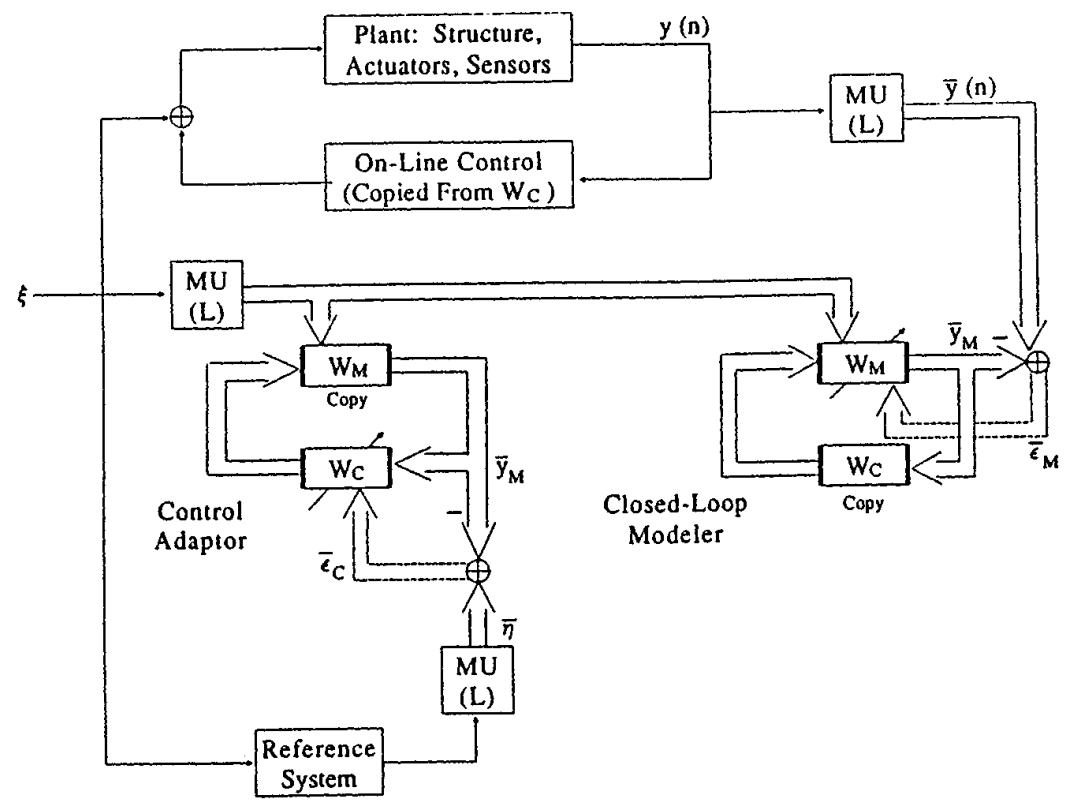

Figure 4. A basic adaptive neural control scheme.

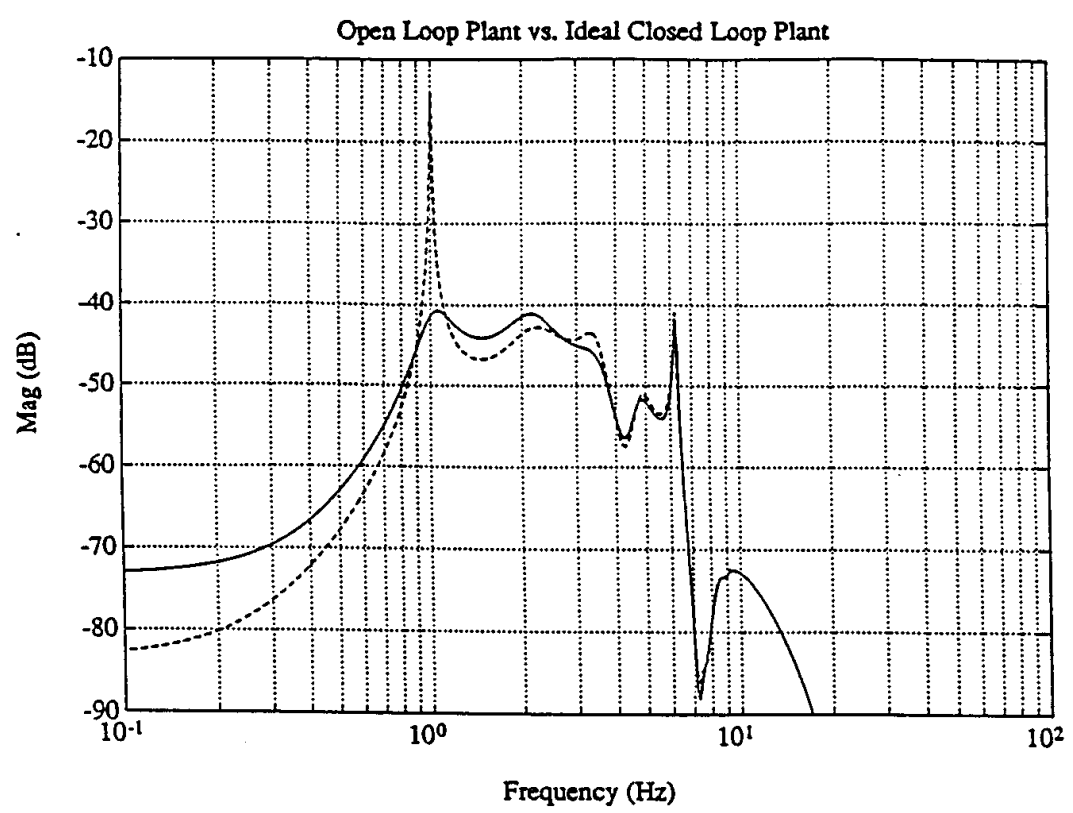

Figure 5. Mini-MAST open-loop against ideal closed-loop plant responses.

In general, the experiments performed by the authors involve multiple inputs and outputs and nearly all involve fairly complex structures with many modes in the frequency band of interest. The laboratory experiments also tested the algorithm under such real-world complications as sensor noise and ambient steady-state disturbances. Summarizing these experience, we can say that reasonably complex multimode systems can be identified with excellent accuracy with convergence times ranging from a few minutes to fractions of a second.

Several replicator units are combined in order to form the ANC system. An ANC performs on-line, simultaneous system identification and adaptively optimized control.
The most basic ANC architecture shown in figure 4 for simultaneously replicating an unknown plant and adapting an output feedback controller so as to match the closedloop input/output characteristics with a prescribed reference system has two parts: (1) the closed-loop modeller and (2) the control adaptor. In figure 4, MU stands for memory units and $\xi$ is for the training signal. The closed-loop modeller uses training signals and the plant sensor output to adapt the weights $w_{M}$, so that the closed-loop is replicated. After convergence $\left(\bar{\varepsilon}_{M} \rightarrow 0\right)$, the modeller output matches the closed-loop system, in effect the modeller identifies the plant within the closed loop. Note that in the modeller, $w_{C}$ is constrained to be equal to the current values being updated 


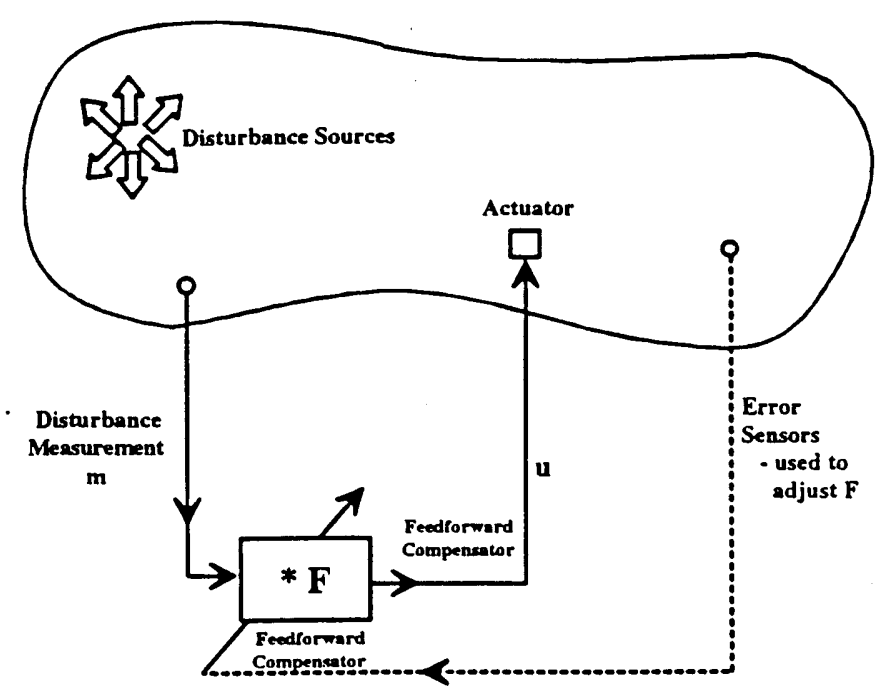

(*denotes convolution)

Once $F$ converges, $m$ directly drives u

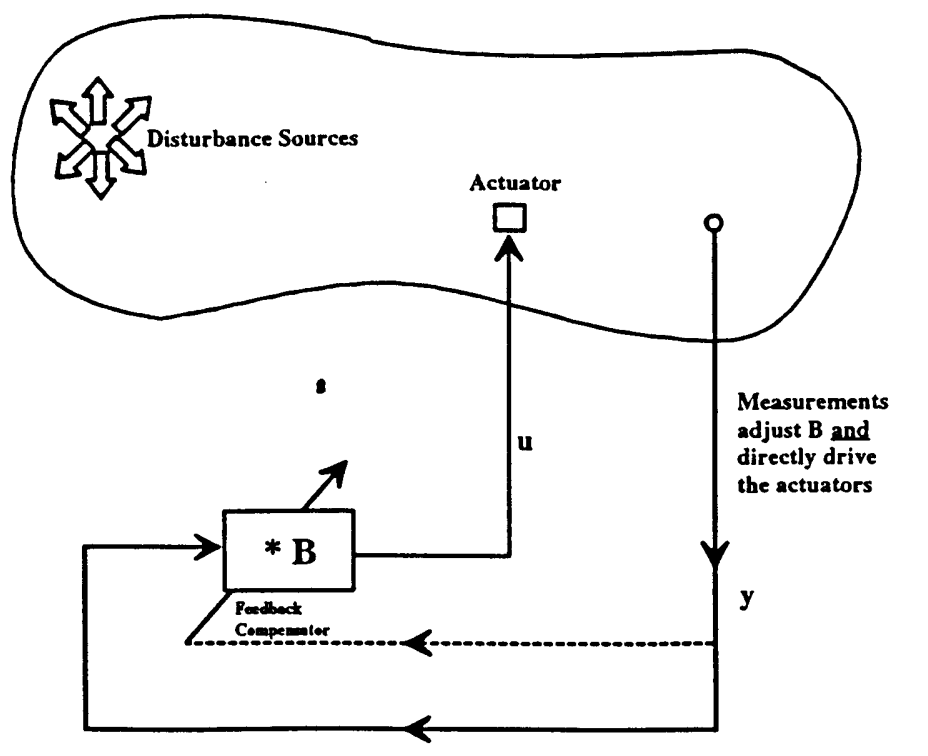

Once B converges, y drives u

\section{Adaptive \\ Feedforward Cancellation}

\section{Adaptive \\ Feedback Control}

- Comparable or superior performance capabilities

- Better transient response

- Can simplify control hardware

- Can handle disturbances that are inaccessible to measurement

Figure 6. Feedforward against feedback ANC architectures.

in the control adaptor. In the control adaptor, $w_{M}$ is a copy of the value currently arrived at by the modeller, while $w_{C}$ is unconstrained. $w_{M}$ is a neural copy of the plant. Thus the control adaptor can, in effect, back-propagate least mean square (LMS) error through the plant to the controller output location. With its internal model of the plant, the adaptor uses the training signal, its own output and that of the reference system to adjust $w_{C}$ so that the reference system is asymptotically replicated. The reference system in the form of model reference adaptive control (MRAC) framework described all desired closed-loop control specifications (e.g. a linear and highly-damped system).

To-date, several demonstrations have been validated for closed-loop adaptive control via ANC. To illustrate the operation of the ANC we consider the adaptive control of the Mini-MAST test facility. The only difference from the set up discussed above is that the accelerometer signals are lowpass filtered to produce approximate rate measurements that are to be used for control feedback. Figure 5 shows the open-loop plant frequency response (from torque wheel to rate measurement) and the reference system frequency response. As indicated, the control objective is simply to attenuate the first bending mode response by a little over $20 \mathrm{~dB}$ while leaving higher frequency dynamics unaltered. The experimental result shows the exact agreement with control objective achieved within $7.5 \mathrm{~s}$ of adaptation.

The above form of ANC addresses the feedback type of control architecture. It is useful to note the two quintessential architectures for adaptive control: feedforward cancellation and feedback control. Figure 6 illustrates these 
two basic disturbance suppression strategies. In feedforward cancellation, the system motion measurements (the error sensor outputs) are used only to adjust the feedforward gain $\mathbf{F}$. Once $\mathbf{F}$ converges, the actuators are driven by the disturbance measurements or a synchronization signal or timing pulse (if the disturbances are periodic). In contrast, adaptive feedback uses the motion measurements not only to adjust feedback gain $\mathbf{B}$ but also to drive the actuators. This is the most suitable architecture for systems wherein disturbances are not accessible to direct measurement.

A large subset of applications, however, involve disturbance sources (e.g. motors, engines, and rotors) that are accessible to direct measurement and, hence, can be suppressed via adaptive feedforward control. The primary input to the neural controller is a synchronization signal taken directly from the vibration source or some indirect disturbance measurement. The neural controller adjusts its parameters, using the outputs of error sensors, (which measures noise or vibration at points where quiescence is desired) and transforms the disturbance measurement into the actuator drive signal, using its identified models of the transfer functions from disturbance measurement and actuator command to error measurement. Once the algorithm has converged, the output of the actuators cancels the disturbance at the locations of the error sensors. The ANC architecture is readily applied to handle this situation. The adaptive neural controller for feedforward is able to simultaneously identify all needed transfer functions and adapt the actuator inputs without interrupting normal operation or injecting an extraneous broadband test signal (dither). Moreover, the adaptive algorithm is fast: with no previous identification completed cancellation is achieved. Thus the neural controller is able to autonomously react to rapid changes in the disturbances or in the system dynamic characteristics.

All experimental results are further validated using a Harris in-house facility, multi-hex prototype experiment (MHPE). The MHPE as shown in figure 7 has the form of a four meter Cassagrain telescope and consists of a base plate (supported by an air bag) connected by a six member truss to the primary mirror support structure, holding a tripod tower which supports the secondary mirror assembly. Disturbances are injected using a proof-mass actuator (LPACT) affixed to the primary mirror support structure. Three additional LPACTs with collocated accelerometers are mounted on the secondary mirror platform to provide actuation and motion sensing. The objective is to suppress vibrational accelerations at the secondary mirror platform that are excited by the forces applied to the primary support structure. Experimental investigations proceeds in several stages, beginning from 'harmonic' or single-tone disturbances to 'broadband' or continuous spectrum disturbances with the following results:

- Single-tone (harmonic) disturbances. Disturbance sources for many applications are dominated by a fundamental harmonic component, where the amplitude may vary over time. Examples include fan noise, automobile engine noise, aircraft cabin noise, etc. In many of these cases the disturbance source is directly accessible to measurement, whether through a

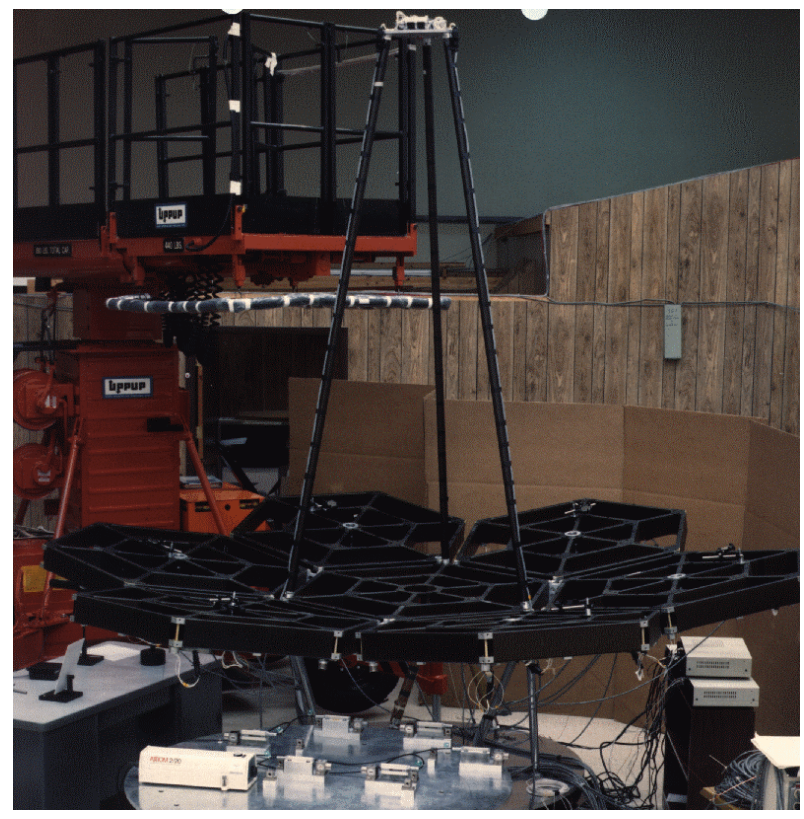

Figure 7. Harris MHPE test facility.

synchronization or timing pulse pick-off directly from the noise source or by use of motion sensors located on or very near the source. The ANC algorithm for harmonic noise cancellation was implemented with a PC interfaced digital signal processors (DSPs) and demonstrated on three entirely different laboratory test stands: an acoustic duct, a test rig for a force cancellation proof mass actuator and the MHPE testbed, a precision optical structure. Starting with no prior identification data, the system converges in a fraction of a minute. Over $20 \mathrm{~dB}$ noise or vibration reduction is achieved in all cases.

- Multi-tone (several harmonics) disturbance. This is a significant feature in most applications (e.g. nonlinearities in engines and motor mounts produce higher harmonics and subharmonics in addition to the fundamental tone produced by the rotary source). In many cases, the multi-tone disturbance is truly periodic. There are other cases where the constituent tones are incommensurate. The ANC system for this case combines a fast neural demodulator unit with an array of single tone cancellors. Over $30 \mathrm{~dB}$ and approximately $45 \mathrm{~dB}$ attenuation is achieved on the three tones. Starting with no prior transfer coefficient information, simultaneous identification and control are achieved in approximately one minute.

- Continuous spectrum disturbances: Broadband disturbances are defined as having continuously distributed power spectral densities. This class of disturbances is a non-negligible component in many applications; e.g. fluid sloshing noise in dish washers and washing machines. In many cases, these disturbances are not accessible to direct measurement so that the feedback control architecture must be used. In other cases, even though the disturbances are amenable to measurement, the broad spectral distribution of the disturbances make a combined feedforward and feedback control architecture the most efficient, robust approach. The ability to implement 
Table 1. Candidate ANC designs on MHPE testbed ('time'-time-domain; 'frequency'-frequency domain; 'time and frequency'—mixed time and frequency; 'SISO'- single-input single-output; 'MISO'-multiple-input single-output).

\begin{tabular}{|c|c|c|}
\hline Program & Configuration & Result \\
\hline $\begin{array}{l}\text { ANC } 1 \\
\text { 'time' }\end{array}$ & $\begin{array}{l}\text { Off-line checkout of delay line replicator } \\
\text { code segments }\end{array}$ & \\
\hline $\begin{array}{l}\text { ANC } 2 \\
\text { 'time' }\end{array}$ & SISO ARMA series-parallel ID & $\begin{array}{l}\text { Used a shaker input, got good match of I/O } \\
\text { behavior using feedback system output }\end{array}$ \\
\hline $\begin{array}{l}\text { ANC } 3 \\
\text { 'time' }\end{array}$ & $2 \times 2$ MIMO ARMA series-parallel ID & $\begin{array}{l}\text { Required } 3-5 \text { min to converge (for } \\
\text { unaccounted time delay) }\end{array}$ \\
\hline $\begin{array}{l}\text { ANC } 4 \\
\text { 'time' }\end{array}$ & $\begin{array}{l}2 \times 1 \text { MISO ARMA ID with algebraically } \\
\text { constrained control (deadbeat control) }\end{array}$ & $\begin{array}{l}\text { Unstable_puzzling because of apparent good } \\
\text { input/output behavior match prior to control. } \\
\text { Note possibility of non-minimum phase } \\
\text { model }\end{array}$ \\
\hline $\begin{array}{l}\text { ANC } 5 \\
\text { 'frequency' }\end{array}$ & $\begin{array}{l}\text { Off-line checkout of FFT/replicator code } \\
\text { segments }\end{array}$ & \\
\hline $\begin{array}{l}\text { ANC } 6 \\
\text { 'frequency' }\end{array}$ & SISO FFT/replicator ID & $\begin{array}{l}\text { Matched primary path well with a } 1 \mathrm{~Hz} \\
\text { resolution }\end{array}$ \\
\hline $\begin{array}{l}\text { ANC } 7 \\
\text { 'frequency' }\end{array}$ & $\begin{array}{l}2 \times 1 \text { MISO ID with FFT/replicator, } \\
\text { control via division of identified primary } \\
\text { and secondary path weights to form FFT } \\
\text { based controller }\end{array}$ & $\begin{array}{l}\text { Distributed } 5-30 \mathrm{~Hz} \text { band, error and drift in } \\
\text { the weights below } 5 \mathrm{~Hz} \text { caused large drifts in } \\
\text { control }\end{array}$ \\
\hline $\begin{array}{l}\text { ANC } 8 \\
\text { 'frequency' }\end{array}$ & $\begin{array}{l}2 \times 1 \text { MISO ARMA ID, control via pre- } \\
\text { filtered replicator adaptation of FFT } \\
\text { controller weights }\end{array}$ & Drifting/unstable controller adaptation \\
\hline $\begin{array}{l}\text { ANC } 9 \\
\text { 'frequency' }\end{array}$ & $\begin{array}{l}\text { SISO FFT weight filter with fixed, user- } \\
\text { selected FRF }\end{array}$ & $\begin{array}{l}\text { Verified ability to implement a specified FRF } \\
\text { with this type of FFT filter }\end{array}$ \\
\hline $\begin{array}{l}\text { ANC } 10 \\
\text { 'time' }\end{array}$ & $\begin{array}{l}2 \times 1 \text { MISO ARMarkov series-parallel } \\
\text { ID, control via explicit FRF computation } \\
\text { and assignment to FFT weight controller }\end{array}$ & $\begin{array}{l}\text { Revealed serious sensitivity to roundoff error } \\
\text { (due to finite match precision) with weight } \\
\text { updates and delay-line filter computations }\end{array}$ \\
\hline $\begin{array}{l}\text { ANC } 11 \\
\text { 'frequency' }\end{array}$ & $\begin{array}{l}\text { SISO ID of a single frequency bucket } \\
\text { using cross and auto spectral density } \\
\text { averaging based on recursive FFT }\end{array}$ & $\begin{array}{l}\text { Computes good results as compared with } \\
\text { spectrum analyser, but would like a little } \\
\text { more speed }\end{array}$ \\
\hline $\begin{array}{l}\text { ANC } 12 \\
\text { 'frequency' }\end{array}$ & $\begin{array}{l}\text { SISO ID of multiple frequency buckets } \\
\text { using ANC } 11 \text { algorithm, estimates } \\
\text { accuracy of each bucket ID, adjusts FFT } \\
\text { control of each bucket independently }\end{array}$ & $\begin{array}{l}\text { Good results, desire increased speed and } \\
\text { decreased complexity. Also, improved rigor } \\
\text { needed in ID (really need twice the resolution } \\
\text { of control FFT in order to improve } \\
\text { reliability) }\end{array}$ \\
\hline $\begin{array}{l}\text { ANC } 13 \\
\text { 'frequency' }\end{array}$ & $\begin{array}{l}\text { SISO FFT replicator with adjustable } \\
\text { learning rate for ID (twice resolution of } \\
\text { control), control algorithm requires no ID } \\
\text { accuracy estimate }\end{array}$ & $\begin{array}{l}\text { Reliable convergence on center of channel, } \\
\text { but poor performance in between }\end{array}$ \\
\hline $\begin{array}{l}\text { ANC } 14 \\
\text { 'time and } \\
\text { frequency' }\end{array}$ & $\begin{array}{l}\text { Replicator with adjustable learning rate } \\
\text { also used for control updates }\end{array}$ & $\begin{array}{l}\text { Reliable convergence, good overall } \\
\text { performance compromise achieved over } \\
\text { whole frequency band }\end{array}$ \\
\hline
\end{tabular}

adaptive feedback and combined feedforward, feedback control architectures is a unique strength of ANC technology. The broadband ANC cancellor was successfully demonstrated in the laboratory using the MHPE testbed. Dozens of resonances near 10 and $15 \mathrm{~Hz}$ were reduced to the instrumentation noise floor within a few minutes.

The above results demonstrate fast, adaptive cancellation for all principal classes of disturbance. When disturbances are accessible to (at least indirect) measurement, a unique feature of the ANC architecture is that it can combine feedforward control with feedback loop. These two forms of control have synergistic benefits. First feedback greatly improves transient response and this permits faster feedforward convergence. Also, closed-loop feedback smooths out the system transfer functions. This allows the feedforward control to track much more rapid system changes, e.g. disturbance frequency variations due to motor acceleration/deceleration. Alternatively, smoother transfer function variations permit a simpler and cheaper feedforward system to be used.
Table 1 shows a complete list of the developed architectures in chronological order. The thirteen major experimental tests that were performed on MHPE testbed using both time- and frequency-domain versions. For details of the analytical and experimental developments, please refer to [18].

The general situation pertaining to adaptive feedforward control is illustrated in figure 8 . The most basic assumption is that disturbance sources are accessible to measurement, either through a sensor very close to the disturbance or through a direct synchronization pick-off signal. This produces a disturbances measurement signal $m(t)$. A second input $z$, to the control system is provided by the set of error sensors which measure system response at locations where quiescence is desired. We considered the class of disturbances consisting of a combination of several sinusoids and assume that the corresponding synch signals are provided. In addition to the adaptation operations per se, there are two ancillary operations that must be performed (assuming $L$ harmonics in the disturbance): (1) using 


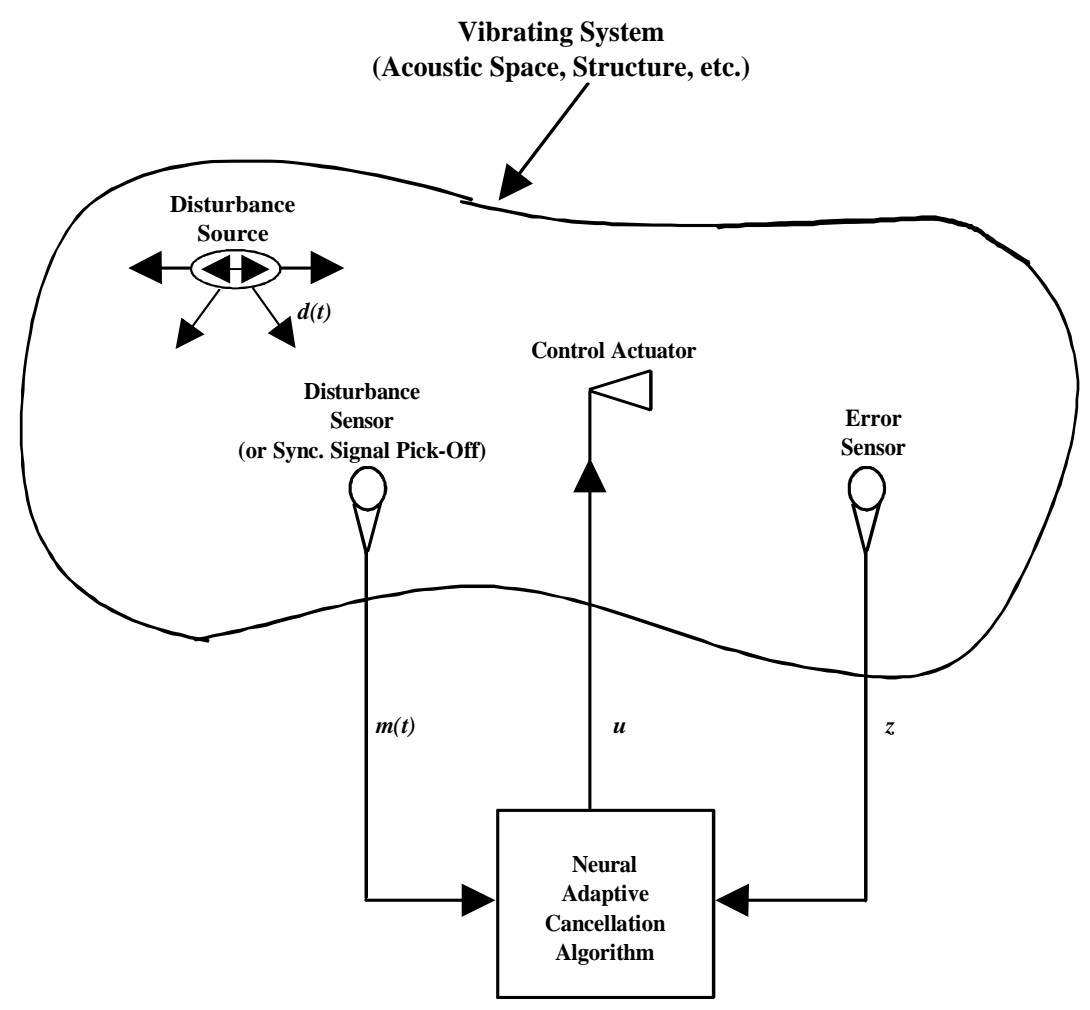

Figure 8. Adaptive feedforward neural control for the ASTREX experiment.

the $L$ synchronization signals from the several harmonic disturbance sources, generate sine waves, both in phase and quadrature at the $L$ frequencies; and (2) given the error sensor outputs, decompose these measurements into the separate components at each of the $L$ frequencies (i.e. demodulate the error measurements).

\section{MIMO feedforward control of tonal disturbances-ASTREX}

As described in the preceding section, extensive results have been achieved for system identification, for adaptive feedback control with a modest number of inputs and outputs and for adaptive feedforward control for singleinput, single-output (SISO) systems. However, there remain many developments of ANC to be completed. For example, although feedforward control was demonstrated for both tonal and continuous spectrum disturbances, only SISO systems have been extensively tested. The most recent work reported here advances ANC development by the experimental demonstrations on multi-input, multi-output (MIMO) feedforward control of tonal disturbances. The MIMO aspect of the problem offers distinct challenges that must be addressed for successful real-world applications. Among the practical benefits being sought are reliable, selfreliant and self-contained vibration isolation for sensitive payloads. The control scheme developed assumes only that the error sensor signals and frequencies of the sinusoids are measured. All system dynamics are determined on line, and the error sensor outputs are to be optimized in a least squares sense following failures of arbitrary subsets of actuators and sensors.

\subsection{ASTREX experiment setup}

The ASTREX test facility (as seen in figure 9) is located at the Air Force Research Laboratory, Kirtland Air Force Base, New Mexico [1,25-27]. The ASTREX structure consists of two major parts: a pivoting test article and vertical pedestal which supports the test article through an air-bearing system. The test structure is designed to accommodate the concepts of a typical space-based beam expander or a space-based radar. The test article's primary truss consists of graphite/epoxy (Gr/Ep) composite modular tubes with aluminum joints. The front section of the test article is connected to the primary truss by a tripod support. The rear section, called tertiary, houses electronics and balances the secondary. On the primary truss, six triangular plates on the front represent the inertia of a $5 \mathrm{~m}$ diameter mirror, and two cylindrical masses on the sides represent the inertia of two tracking telescopes. The total weight of the test article is about 10000 pounds and it floats on the air-column. The test article is equipped with a variety of actuators and sensors. The actuators installed on the structure include: a reaction wheel, twelve reaction control system (RCS) thrusters, three linear precision actuators (LPACTs), four control moment gyroscopes (CMGs) and three advanced composites with embedded sensors and actuators (ACESA) smart struts with embedded piezoelectric actuators [28]. In addition, four 100 pound shakers are available for system identification and modal survey.

A summary block diagram of the experimental demonstration is shown in figure 10 . It consists of six major components: the ASTREX structure, the ACESA secondary mounting struts and electronics, the LPACTS and 


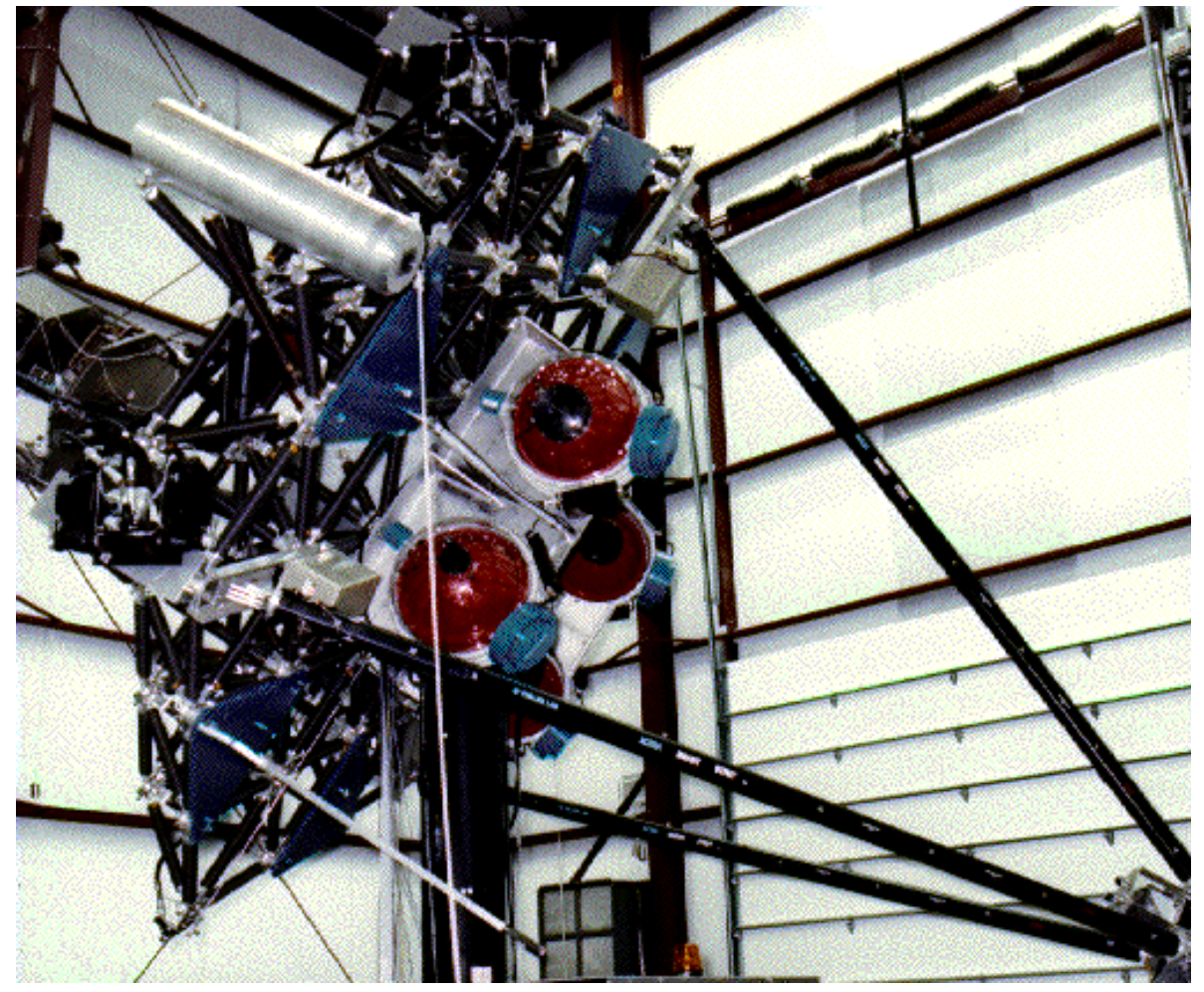

Figure 9. AFRL ASTREX test facility.

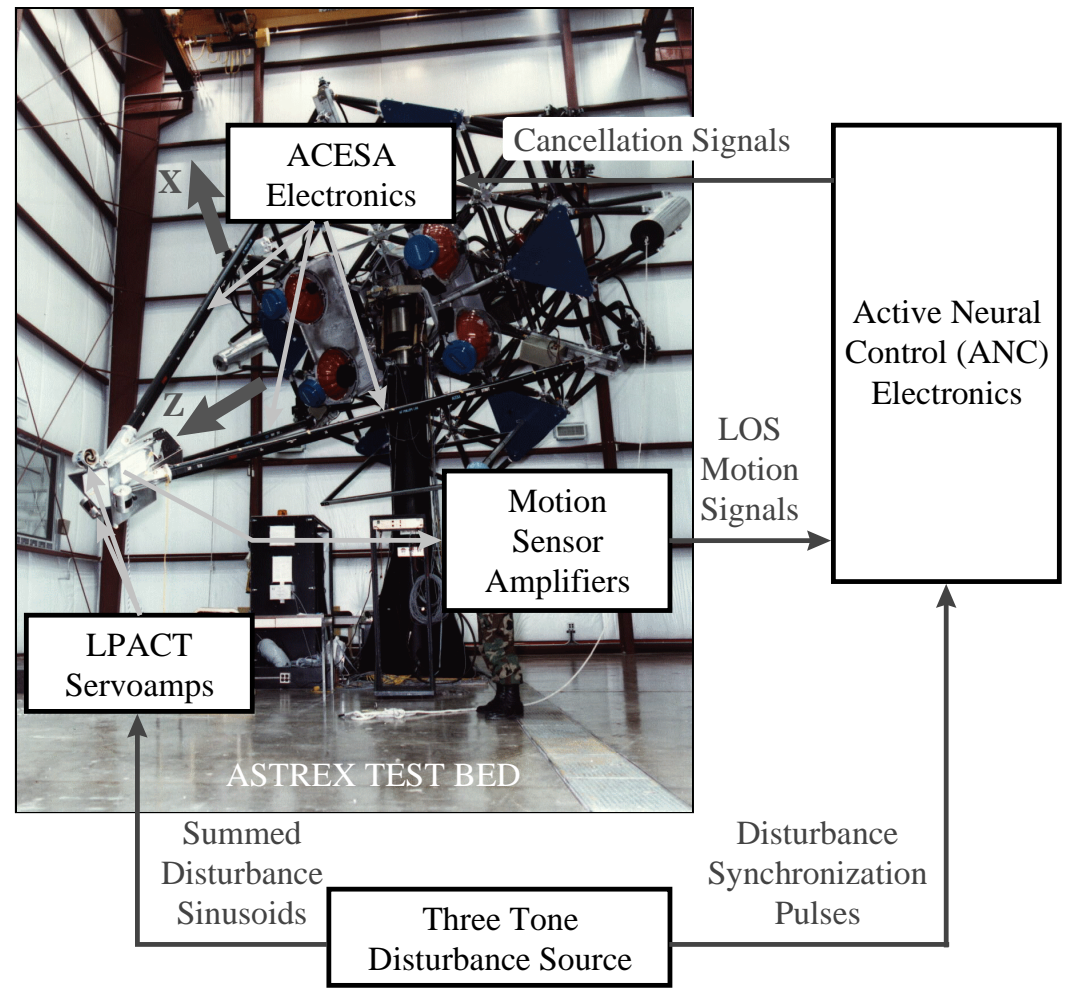

Figure 10. Block diagram of the ASTREX experimental demonstration.

servoamps, the three-tone disturbance source, the motion sensors and amplifiers, and the ANC control system. Three sinusoidal disturbances are generated, scaled and summed in the disturbance source block. These sums are fed to two of the three LPACTs on the secondary tower of the ASTREX test bed as system disturbances. Meanwhile, three pulse trains, synchronized with each of the three sinusoids, are passed to the ANC controller. Thus, the controller 


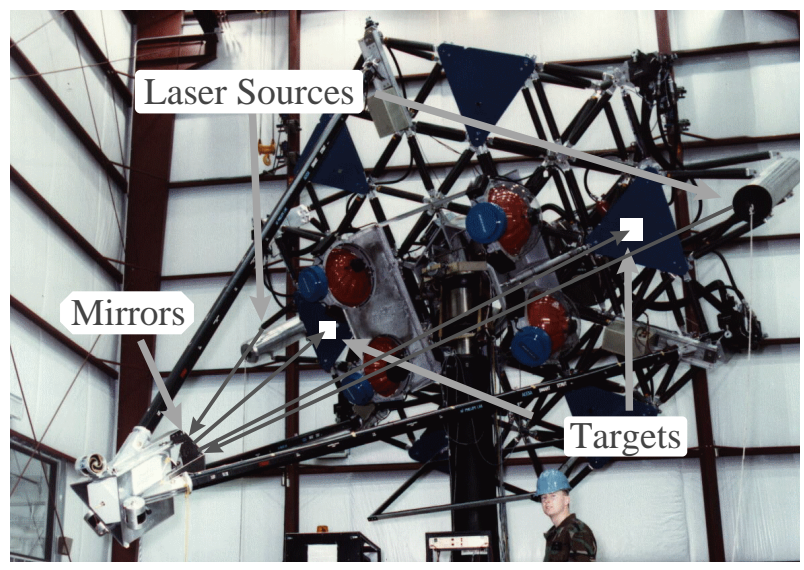

Figure 11. Performance visualization system for the ASTREX demonstration.

knows the frequency, but not the amplitude or phase of the disturbance inputs to the LPACTs. The controller synthesizes six canceling control signals to feed to the ACESA struts, one for each bending axis of the three struts. The resulting motion of the structure, which is to be canceled out, is sensed and fed to the ANC controller as a training signal.

The demonstration emulates a precision optical system that is disturbed by independent disturbances such as might originate from three CMGs with slight imbalances. The motion of key optical subsystems that contribute to lineof-sight (LOS) error are sensed and canceled by the ANC controller using actuators capable of controlling the relevant substructures (i.e. ACESA struts). In addition to the components shown in figure 10, an independent scoring system shown in figure 11 was installed to enable visual observation of the vibration cancellation. Two laser sources are mounted near the mass of telescope trackers. These aim at two small mirrors mounted on the secondary assembly. The laser beams are reflected on to paper targets mounted on the primary structure. This provides complete monitoring of the effects of the secondary mirror assembly on the LOS performance and is entirely independent of the sensors used for training the ANC.

The ACESA struts were developed by TRW [28]. The interface to the ANC system is via six analog inputs, which command the three struts, each in two bending axes. The struts are labeled for convenience as the 12:00 strut, the 4:00 strut and the 8:00 strut according to their positions as viewed by an observer looking in the $Z$-direction (down the boresight from the secondary mirror assembly towards the primary). Each strut has two inputs: one to cause motion of the secondary in the $X$-direction, and one to cause motion in the $Y$-direction. For safety reasons the inputs were limited to $\pm 3 \mathrm{~V}$ to prevent damage to the structure or struts.

The motion sensing for ANC adaptation consisted of three angular velocity sensors mounted near the mirrors on the secondary. These were insensitive to translation of the tower and provided a much better indication of the effect of the secondary on the visually observed spot motion of the laser beams on the targets. The velocity sensors had sensitivities of approximately $400 \mathrm{mV} \mathrm{s} \mathrm{rad}-1$.

The disturbance generation system works according to the block diagram shown in figure 12. Three signal generators

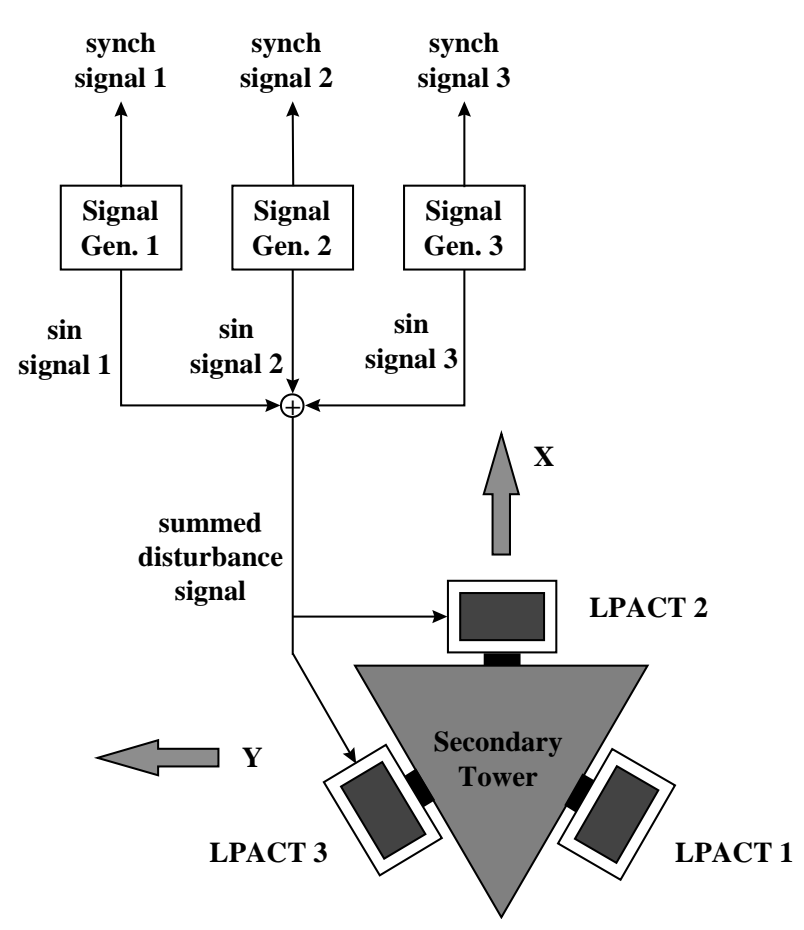

Figure 12. The disturbance generation system for the ASTREX demonstration.

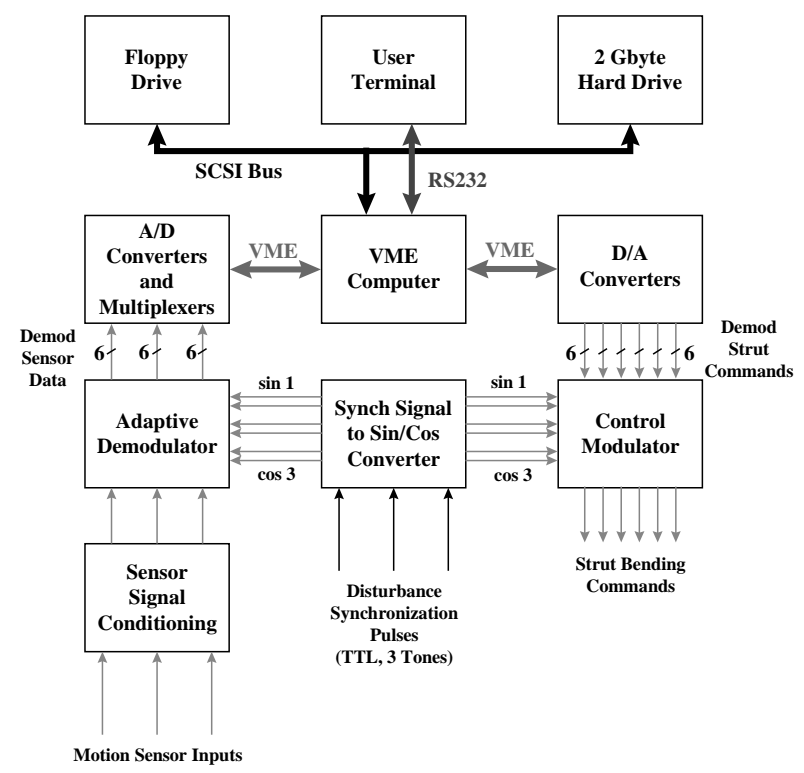

Figure 13. Block diagram of the ANC system.

provide individually adjustable sinusoids, which are summed and input to the LPACTs used for disturbance generation. The summing function is provided by a simple op-amp circuit. The same disturbance signal is input to LPACT servoamps. The LPACTs provide approximately $10 \mathrm{lb}$ maximum output for a maximum input of approximately $5 \mathrm{~V}$ at $10 \mathrm{~Hz}$. Slightly more output is available at higher frequencies.

The ANC system has the structure shown in the block diagram of figure 13. Except for the VME computer peripherals (i.e. disk units and terminal), the whole assembly fits in a VME card cage. This implementation of the ANC 


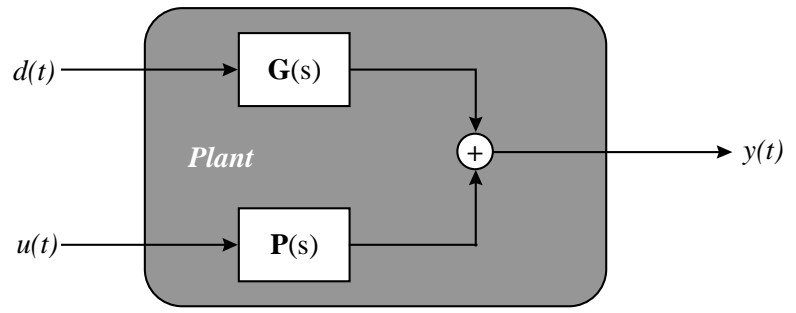

Figure 14. The basic system notation.

system is based on the observation that if the control system and the disturbance inputs are the sum of three sinusoids, then the motion sensor outputs must also be the sum of three sinusoids. This is certainly true if the system is linear. Furthermore, the required cancellation signals must be the sum of three sinusoids in this case. Note that any sum of three sinusoids is specified by the six coefficients of the cosines and sines of which it is comprised. The ANC cancellation system is built to determine and operate on these coefficients. The three disturbance synchronization signals are converted in the largest analog circuit card into sine and cosine signals at each of the three disturbance frequencies. These are used as the fundamental basis functions to process both the motion sensor signals and the control commands. The motion sensor inputs are amplified and low-pass filtered and then are fed into the adaptive demodulators. The demodulators determine what combination of the three sine and cosine signal pairs are in each of the three motion sensor signals. For each of the three sensors there are six coefficients which are then fed into the analogue-to-digital (A/D) converters. It takes approximately $20 \mathrm{~s}$ for these signals to reach their final values once a new set of demodulated strut commands are issued by the VME computer.

The A/D converter system, residing on one master A/D card and two slave multiplexer cards, converts the 18 coefficients used by the cancellation algorithm to the VME computer for processing. The resolution is 12 bits. The 12 bit D/A converters, residing on two VME cards, issue six commands for each of the six strut bending axes. The six commands per axis are the proportions of the three pairs of sine or cosine signals which are desired in the strut command for a single strut axis. The modulator multiplies the six coefficients per channel times the appropriate sine and cosine signals to compute the command for each strut's two bending axes.

\subsection{ANC control algorithm}

The general situation pertained to adaptive feedforward control is illustrated in figure 14. The most basic assumption is that disturbance (i.e. $d(t))$ frequencies are accessible to measurement (i.e. $\omega_{i}, i=1, \ldots, n$ ), either through a sensor very close to the disturbance or through a direct synchronization pickoff signal. A second input to the control system is provided by the set of 'error sensors' which measure system response (i.e. $y(t))$ at locations where quiescence is desired. The disturbance to the system and the control signal

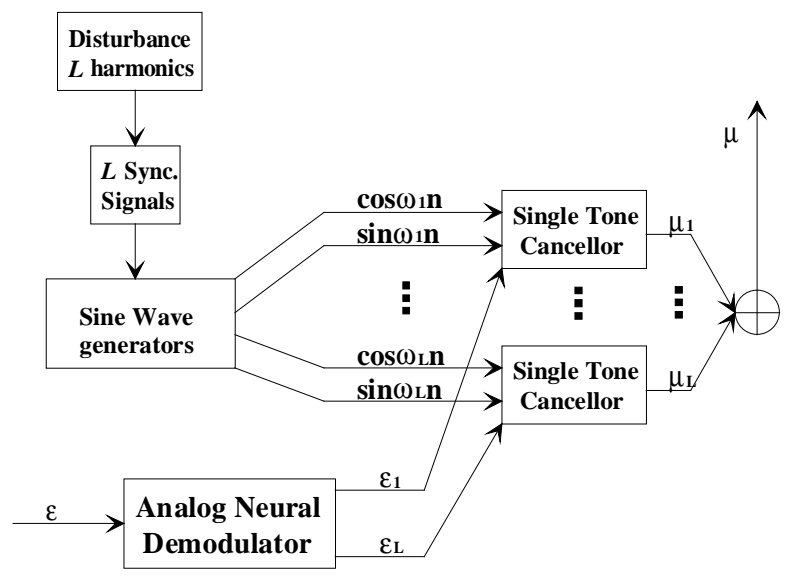

Figure 15. ANC controller block diagram for multi-tone.

(i.e. $u(t))$ are assumed to be the sum of $n$ sinusoids. In brief,

$$
\begin{aligned}
& d(t)=\sum_{i=1}^{n} \operatorname{Re}\left(d^{(i)} \mathrm{e}^{-\mathrm{j} \omega_{i} t}\right) \\
& u(t)=\sum_{i=1}^{n} \operatorname{Re}\left(u^{(i)} \mathrm{e}^{-\mathrm{j} \omega_{i} t}\right)
\end{aligned}
$$

where $\operatorname{Re}(\cdot)$ denotes the real part of a complex argument. Control is accomplished individual 'epoch' consisting of the application of a set of complex weights to be used in constructing the control signal, followed by a waiting period to allow a new steady state to be achieved. The control system then records the complex components of the various sinusoids present in the output, and computes a new set of complex control weights. The analog control modulator constructs the control input sum from the commanded complex weights and the sinusoids provided. The analog demodulator decodes what combination of complex components of each sinusoid are present in the error measurements. Figure 15 show the block diagram of the ANC system for multi-tone vibration cancellation.

Once a steady state has been reached, it is clear from basic system theory that the complex components present in the output at the end of the $k$ th epoch can be written, in the absence of noise, simply as

$$
\begin{aligned}
y(t) & =\sum_{i=1}^{n} \operatorname{Re}\left\{G\left(-\mathrm{j} \omega_{i}\right) d^{(i)} \mathrm{e}^{-\mathrm{j} \omega_{i} t}+P\left(-\mathrm{j} \omega_{i}\right) u^{(i)} \mathrm{e}^{-\mathrm{j} \omega_{i} t}\right\} \\
& =\sum_{i=1}^{n} \operatorname{Re}\left\{\left(g^{(i)}+P^{(i)} u^{(i)}\right) \mathrm{e}^{-\mathrm{j} \omega_{i} t}\right\} \\
& =\sum_{i=1}^{n} \operatorname{Re}\left\{y^{(i)} \mathrm{e}^{-\mathrm{j} \omega_{i} t}\right\}
\end{aligned}
$$

where $g^{(i)} \equiv G\left(-\mathrm{j} \omega_{i}\right) d^{(i)}, P^{(i)} \equiv P\left(-\mathrm{j} \omega_{i}\right)$ and $y^{(i)}=$ $g^{(i)}+P^{(i)} u^{(i)}$. The goal is to set the $y^{(i)}$ to zero for each of the $n$ tone (i.e. $i=1.2 \ldots, n$ ). Note that the problem for each of the three tones is entirely independent. It is evident from this expression that the various frequencies may be considered independently and that the 'plant' to be controlled, as viewed from the digital controller is the static, 
Table 2. Test cases on the ASTREX facility.

\begin{tabular}{llr}
\hline Case & $\begin{array}{l}\text { Active } \\
\text { actuators }\end{array}$ & $\begin{array}{l}\text { Convergence } \\
\text { time (min) }\end{array}$ \\
\hline 1 & $1,2,3,4,5,6$ & 10 \\
2 & $1,2,4,5,6$ & 5 \\
3 & $1,2,4,5$ & 5 \\
\hline
\end{tabular}

linear, complex mapping shown in equation (3). In the above, one usually refers to $g^{(i)}$ as the 'direct path' dynamics since it specifies the open-loop transfer function from $d(t)$ to $y(t)$. Analogously, $P^{(i)}$ is termed the 'secondary path' dynamics since one might view it as the transfer function from $u(t)$ which is a 'secondary disturbance source', to $y(t)$. In the absence of noise equation (3) makes it obvious that our goal is to construct a control weight such that

$$
u^{(i)}=-P^{(i)^{+}} g^{(i)}
$$

where $(\cdot)^{+}$denotes the group generalized inverse. This choice minimizes the output in a least-squared error sense. The problem addressed herein is how to synthesize the above optimal actuator commands without a priori knowledge of $g^{(i)}$ or $P^{(i)}$ and to implement and demonstrate such a controller in real hardware under numerous practical constraints. With regard to the digital algorithm indicated in figure 15 , a wide selection of algorithms have been devised and experimentally tested on a simpler testbed as listed in table 1 . The version utilized here on the ASTREX test article consists of two parts.

- A set of components from the ANC architecture designed to simultaneously identify $g^{(i)}$ and $P^{(i)}$ given the past histories of $y^{(i)}$ and $u^{(i)}$. On-line Kalman filtering of the measurements is used [29,30], considering $g^{(i)}$ and $P^{(i)}$ to be a single parameter vector to be estimated. Please see [18] for the details of the Klaman filter cancellation algorithm.

- Computation of the optimum control as in equation (4) via the singular value decomposition, with an appropriate tolerance for robust determination of the numerical rank.

Note that equation (4) allows optimum inversion even when $P^{(i)}$ is singular or changes rank owing to actuator or sensor failures. Thus the neural algorithm cannot only 'track' smooth changes in system dynamics, it can achieve the greatest possible degree of control performance recovery following sensor-to-actuator hardware degradation or catastrophic failures.

\subsection{Experimental results}

In summary, the control problem posed for ASTREX was to nullify the three components of line of sight (LOS) error using the six bending motion control channels of the active struts. Thus, the system of equation (4) has six control inputs and three performance outputs. The ANC hardware interfaced with the active strut electronics was tested on the ASTREX facility. In these tests, the disturbance had three harmonics at $10.15,10.625,12.725 \mathrm{~Hz}$ and three basic cases were considered as indicated in table 2 . Note that the convergence

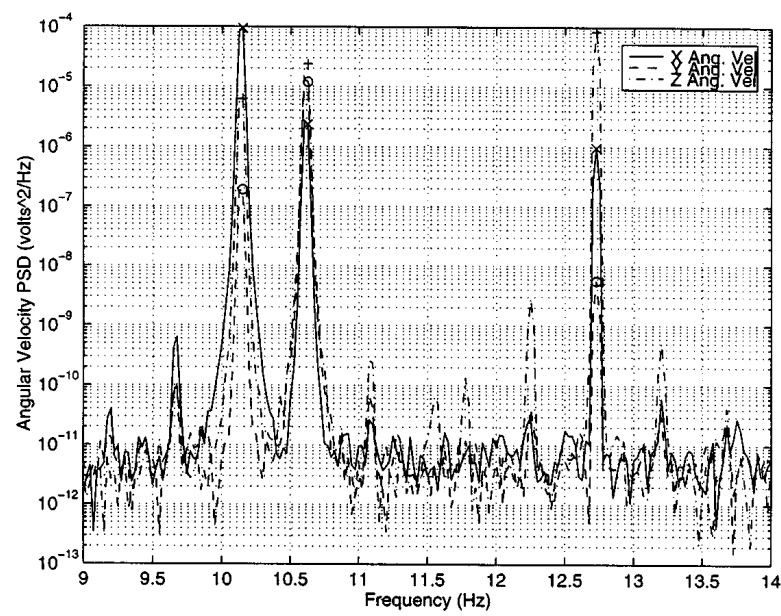

Figure 16. Open loop accelerometer PSDs.

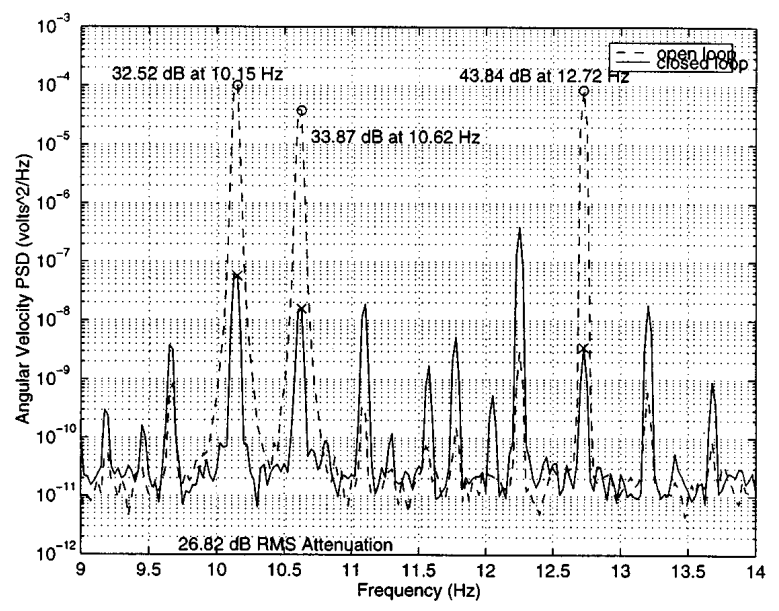

Figure 17. Case 1, closed loop angular velocity PSDs.

time is proportional to the number of actuators and the time required to reach steady state after application of a control signal.

Open-loop results are shown in figure 16. There are the power spectral densities (PSDs) of the voltages, after amplification, of the three angular velocity sensors mounted on the tower. The additional spurs at $12.25 \mathrm{~Hz}$ are due to small nonlinearities in the disturbance system, which cause mixing of the frequencies. Case 1 is intended to show convergence to the optimal feedforward controller beginning with a blank slate (i.e. starting with no modeling information and all neural weights zero). Figure 17 shows the open-loop performance as the sum of the PSDs of the separate motion sensors and the corresponding closed-loop responses after 10 min of convergence time. The ANC algorithm achieved excellent cancellation of the tones: $33 \mathrm{~dB}$ at $10.15 \mathrm{~Hz}$, $34 \mathrm{~dB}$ at $10.625 \mathrm{~Hz}$, and $44 \mathrm{~dB}$ at $12.725 \mathrm{~Hz}$. Since our initial algorithm emphasizes identification accuracy without attempting to optimize computational efficiency, all cases in table 1 required 5 to 10 min to converge to within a few per cent of the final controllers. In case 1 , the system converged to a response giving $27 \mathrm{~dB}$ RMS attenuation over the frequency band of interest. 


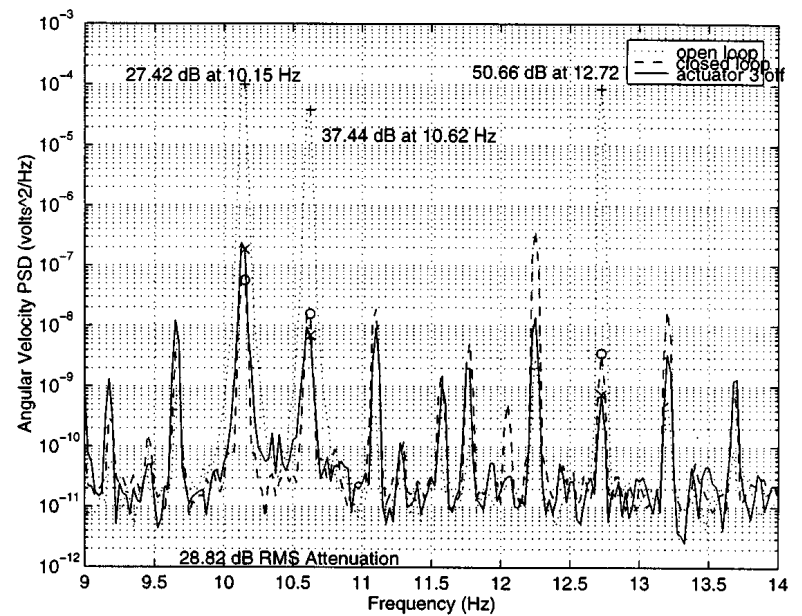

Figure 18. Case 2, closed loop angular velocity PSDs-actuator 3 disengaged.

The remaining cases demonstrate the ANC's capability to recover performance when actuator failures are simulated by disengaging subsets of actuator channels. We progressively disengage more and more actuator channels until there are too few left to achieve full cancellation authority of all disturbance frequencies. Figure 18 shows that happens when actuator 3 is disengaged. The adaptive algorithm immediately detected a change in the plant and reconverged in 5 min with $29 \mathrm{~dB}$ RMS attenuation. Note that since the controller is feedforward, instability cannot result, rather there is a sudden degradation of performance following removal of actuator 3 . The ANC system then reconfigures the control to restore performance to the maximal possible extent. Similarly figure 19 shows what happens when a second actuator, actuator 6, is also disabled. Again, the reconverged response with $27 \mathrm{~dB}$ RMS attenuation shows essentially no performance degradation relative to case 1 . All of these results are fully consistent with the least-squares solutions to the feedforward control problem given the number of actuators that are operational. In all cases, regardless of the number of actuators that were operational, the control adaptation proceeded autonomously with no prior modeling information or human intervention.

\section{Conclusion}

In this paper we have provided a brief review of our analytical and experimental work in the development of the ANC architecture. Further, we have highlighted the most recent demonstrations of ANC for MIMO feedforward control of tonal disturbances on the ASTREX test facility. These preliminary test results demonstrated the capability of ANC to accomplish partial to full controller recovery following the simulated failure of up to one third of the control actuators. In addition, the system developed used an architecture that combined analog and digital ANC elements. This resulted in the very low throughput required. Further testing efforts on the ASTREX facility will concentrate on streamlining the current algorithms so as to significantly increase convergence speed and be ready for flight demonstration. The next step in the development of ANC technology should be to

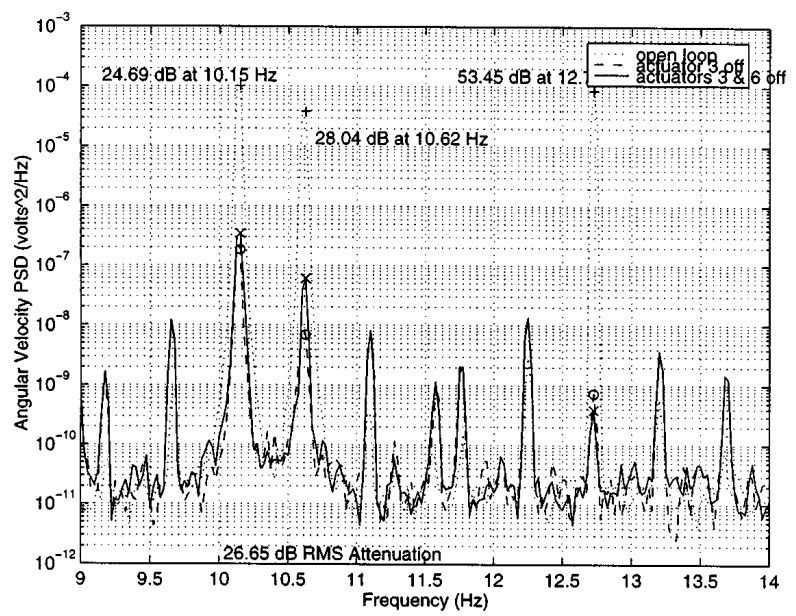

Figure 19. Case 3, closed loop angular velocity PSDs-actuators 3 and 6 disengaged.

demonstrate broadband adaptive cancellation using a small flight-like processor such as the active control patch. This would give potential users confidence that the technology could be integrated into their existing or planned systems with little overhead in terms of cost, volume, weight, or power.

\section{References}

[1] Abhyankar N, Ramakrishnan J, Byun K, Das A, Cossey F and Berg J 1992 Modeling, system identification, and control of ASTREX Proc. NASA/DoD Control Structure Interaction Technology Conf. (Lake Tahoe, NV, March 1992)

[2] Rauch H 1995 Autonomous control reconfiguration IEEE Control Systems 15 37-48

[3] Yen G 1995 Autonomous neural control in flexible space structures Control Eng. Practice 3 471-83

[4] Da R and Lin C 1995 Failure detection and isolation structure for global positioning system autonomous integrity monitoring Proc. AIAA Guidance, Navigation and Control Conf. (San Diego, CA, March 1995)

[5] Rauch H 1994 Adaptive control and fault accommodation using neural network Proc. Yale Workshop on Adaptive and Learning Systems (New Haven, CT, June 1994)

[6] Napolitano M, Naylor S, Neeppach C and Casdorph V 1995 On-line learning nonlinear direct neuro-controllers for restructurable control systems Proc. AIAA Guidance, Navigation and Control Conf. (San Diego, CA, March 1995)

[7] Yen G 1995 Reconfigurable learning control in large space structures IEEE Transactions on Control Systems Technology 2 362-70

[8] Antsaklis P and Passino K 1993 An Introduction to Intelligent and Autonomous Control (Berlin: Kluwer)

[9] White D and Sofge D 1992 Handbook of Intelligent Control-Neural, Fuzzy, and Adaptive Approaches (New York: Van Nostrand Reinhold)

[10] Troudet T, Garg S and Merrill W 1991 Neural network application to aircraft control system design Proc. AIAA Guidance, Navigation and Control Conf. (New Orleans, LA, August 1991)

[11] Narendra K and Balakrishnan J 1997 Adaptive control using multiple models IEEE Transactions on Automatic Control 42 171-87

[12] Antsaklis P 1995 Intelligent learning control IEEE Control Syst. 15 5-8 
[13] Hyland D 1991 Neural network architecture for on-line system identification and adaptively optimized control Proc. IEEE Conf. on Decision and Control (Brighton, UK, December 1991)

[14] Hyland D and King J 1992 Neural network architectures for stable adaptive control, rapid fault detection and control system recovery Proc. AAS Guidance Control Conf. (Keystone, CO, February 1992)

[15] Hyland D 1993 Adaptive neural control architecture-a tutorial Proc. Industry, Government and University Forum on Adaptive Neural Control for Aerospace Structural Systems (Melbourne, FL, August 1993)

[16] Mead C 1989 Analog VLSI and Neural Networks (New York: Addison-Wesley)

[17] Hootman J 1989 Special issue on silicon neural networks IEEE Micro. 9 5-76

[18] Davis L and Hyland D 1996 Adaptive neural control for space structure vibration suppression AFPL Technical Report USAF Phillips Laboratory, Kirtland AFB, NM April 1996

[19] Juang J, Phan M, Horta L and Longman R 1991 Identification of observer/Kalman filter Markov parameters: theory and experiments Proc. AIAA Guidance, Navigation and Control Conf. (New Orleans, LA, August 1991)

[20] Phan M 1993 New concepts in adaptive neural control Proc. the Industry, Government and University Forum on Adaptive Neural Control for Aerospace Structural Systems (Melbourne, FL, August 1993)

[21] Pappa R and Miserentino R 1989 Mini-MAST testbed user's guide NASA Technical Report NASA Langley Research Center, Hampton, VA, March 1989
[22] Smith-Taylor R 1991 The controls structures interaction guest investigator program- an overview and experimental results of Phase I Proc. AAS Guidance Control Conf. (Keystone, CO, February 1991)

[23] Hyland D, Das A, Yen G and Davis L 1996 Autonomous neural control for spacecraft vibration suppression Proc. AIAA Guidance, Navigation and Control Conf. (San Diego, CA, July 1996)

[24] Hyland D 1993 Neural network system identification NASA Technical Report, NASA Langley Research Center, Langley, VA, October 1993

[25] Abhyankar N 1993 A brief description of ASTREX AFPL Technical Report, USAF Phillips Laboratory, Edwards AFB, CA, April 1993

[26] Ramakrishnan J, Hu A and Vander Voort R 1991 Identification experiments on ASTREX Proc. AIAA Guidance, Navigation and Control Conf. (New Orleans, LA, August 1991)

[27] Iyer A 1995 Model reduction and control of ASTREX using stable fractions 1994 Proc. IEEE Conf. on Decision and Control (Lake Buena Vista, FL, December 1994)

[28] Bronowicki A J 1993 Advanced composites with embedded sensors and actuators (ACESA) AFPL Technical Report USAF Phillips Laboratory, Edwards AFB, CA, July 1993

[29] Kirk D 1985 Optimal Control Theory-An Introduction (New York: Addison-Wesley)

[30] Thornton C and Bierman G 1978 Filtering and error analysis via the factorization IEEE Transactions on Automatic Control 23 901-7 